\section{Molecular Syndromology}

Mol Syndromol 2018;9:225-227

DOI: $10.1159 / 000492608$
Accepted: July 11, 2018

by M. Schmid

Published online: August 24, 2018

\title{
The Right Gene, Expressed at the Wrong Time, or at the Wrong Place
}

In medical genetics, clinically relevant mutations are usually thought to damage a gene or to alter its copy number. Changes in sequence, or structural genome variations, not affecting the coding sequence of a gene are generally considered not to be clinically relevant. Nevertheless, chromosomal breakpoints, copy number variations (CNVs), or single nucleotide variations (SNVs) outside the coding sequence of plausible candidate genes have been observed in some patients with dominant developmental disorders [Krebs et al., 1997; Klopocki et al., 2011; Liskova et al., 2018]. Such variations do not alter the functional properties of the encoded proteins. It is conceivable, however, that they may provoke clinical phenotypes by either changing the level of gene expression or by causing ectopic gene expression. Exactly how this may come about is poorly understood. By combining genotypic and phenotypic information on patients with detailed studies of altered development in model organisms into which such variations have been introduced, a few gaps in our understanding have recently been filled.

In patients with acrocallosal syndrome or craniosynostosis of the Philadelphia type, duplications at the $\mathrm{IHH}$ locus were detected [Yuksel-Apak et al., 2012; PaumardHernández et al., 2015]. Since the patient with craniosynostosis with the duplication encompassing the $\mathrm{IHH}$ regulatory region, the $I H H$ encoding gene was thought to be involved in cranial and skeletal development [PaumardHernández et al., 2015]. In mice, the duplications affected conserved noncoding elements, which drove reporter gene expression according to a pattern similar to that of

\section{KARGER}

(C) 2018 S. Karger AG, Basel

E-Mail karger@karger.com

www.karger.com/msy wild-type $I H H$ expression [Klopocki et al., 2011]. The authors concluded that such duplications provoke misexpression and/or overexpression of the $\mathrm{IHH}$ gene and thus affect regulatory signaling networks during digit and cranial development [Klopocki et al., 2011]. In mice, Ihh positively regulates intramembranous ossification, and loss of Ihh reduced Bmp expression within the cranial bones [Lenton et al., 2011]. Ihh is mainly expressed in prehypertrophic and hypertrophic chondrocytes of developing endochondral bones, where it synchronizes chondrogenesis and osteogenesis during endochondral ossification by controlling chondrocyte proliferation and differentiation as well as specification of bone-forming osteoblasts [Ohba, 2016]. IHH directly promotes chondrocyte propagation and forms a negative feedback loop with parathyroid hormone-related protein $(\mathrm{PTHrP})$ to maintain the growth plate length. In addition, IHH activity is required for the specification of progenitors into osteoblast precursors [Ohba, 2016]. At the epiphysis, the thyroid hormone fine tunes levels of IHH and thereby controls the transit of proliferating immature chondrocytes into mature hypertrophic chondrocytes to eventually become osteoblasts [Aghajanian et al., 2017]. While RUNX2 is the master regulatory transcription factor controlling osteogenesis, it is itself regulated by IHH through concomitant inhibition of GLI3-repressor formation and activation of downstream targets [Veistinen et al., 2017]. This suggests that $I H H$ is a key element in a regulatory feedback circuit controlling skull osteogenesis and suture patency [Veistinen et al., 2017]. Together with other

Martin Poot

Department of Human Genetics, University of Würzburg Biozentrum, Am Hubland DE-97074 Würzburg (Germany)

E-Mail Martin_Poot@hotmail.com 
genes, $\mathrm{IHH}$ thus forms a functional network that regulates ossification [Poot, 2018]. Perturbation of IHH function within this gene network may result in skeletal dysmorphologies and craniosynostosis.

Recently, transgenic reporter and genome-editing experiments in mice showed that $\mathrm{IHH}$ expression is regulated by a cluster of conserved noncoding elements consisting of at least 9 enhancers, each with individual tissue specificity in the digit anlagen, growth plates, cranial sutures, and fingertips [Will et al., 2017]. These enhancers function additively as engineered consecutive deletions revealed distinct patterns of growth defects of the skull and the long bones. $\mathrm{IHH}$ duplications, in contrast, not only caused dose-dependent upregulation, but also misexpression of the $\mathrm{IHH}$ gene. The latter led to abnormal phalanges, fusion of sutures, and syndactyly. These findings demonstrate that precise spatiotemporal control of developmental gene expression in the skeletal and the cranial bones is achieved through complex multipartite enhancer ensembles. Alterations in the composition of such clusters by structural variation may provoke gene misexpression and eventually dysmorphic disorders.

Also a specific form of autosomal-dominant corneal endothelial dystrophy, known as posterior polymorphous corneal dystrophy (PPCD), may be due to ectopic gene expression [Liskova et al., 2018]. The clinical course of this disorder is highly variable, ranging from asymptomatic changes of the corneal endothelium, up to blurred and even complete loss of vision. In the latter case, vision can only be restored by corneal transplantation. In a large 9-generation family with 18 living and 19 deceased PPCD patients, neither Sanger sequencing of established PPCDassociated genes, including OVOL2, nor whole exome sequencing revealed any likely disease-associated SNVs. Linkage analysis of 9 patients and 7 unaffected siblings showed linkage for a locus spanning $19 \mathrm{Mbp}$ within region $8 \mathrm{q} 22.3 \mathrm{q} 24.12$, with a maximum LOD score of 4.38 . Whole genome sequencing of 4 distantly related patients and filtering SNVs for this locus turned up 2 intergenic SNVs and 1 cosegregating variant within intron 1 of the GRHL2 gene. This SNV was also found in 3 other unrelated PPCD families and 2 more SNVs in the same intron of GRHL2 in 2 other unrelated PPCD families. In human embryonic stem cells, adult dermal fibroblasts, and normal human epidermal keratinocytes, these SNVs in intron 1 of GRHL2 showed histone 3 methylation and acetylation marks and DNase I hypersensitivity consistent with open and transcriptionally active chromatin. According to MatInspector and AliBaba 2.1, all PPCD-associated GRHL2 SNVs were predicted to result in a gain or loss of binding of at least 1 transcription factor expressed in the corneal endothelium [Grabe, 2002; Cartharius et al., 2005]. Each of the 3 GRHL2 PPCD-associated SNVs showed significantly increased promoter activity in promoter-less firefly luciferase reporter vectors transfected into HEK293 cells. Thus, these PPCD-associated SNVs of intron 1 of GRHL2 enhanced the transcription of their target gene.

Previously, mutations leading to loss of 1 allele of GRHL2 have been found in autosomal-dominant nonsyndromic hearing loss (DNFA28; MIM:608641) and homozygous missense mutations have been associated with autosomal recessive ectodermal dysplasia with hearing loss (ECTDS; MIM:616029) [Van Laer et al., 2008; Vona et al., 2013; Petrof et al., 2014; Kim et al., 2015]. While mutations within the coding sequence or loss of 1 allele of GRHL2 are linked to hearing loss, upregulation and ectoptic expression of GRHL2 are associated with PPCD without hearing impairment [Liskova et al., 2018]. Hence, a specific and novel mechanism for noncoding GRHL2 SNVs in causing PPCD has to be conceived.

ZEB1 maintains human corneal endothelial cells in the normal state by promoting their epithelial-to-mesenchymal transition. GRHL2 and OVOL2 are transcriptional repressors of $Z E B 1$, but $Z E B 1$ can also repress the expression of both of these genes. This mechanism maintains corneal endothelial cells in the healthy cellular state. In PPCD, mutations involving loss of 1 allele or causing reduced expression of $Z E B 1$, and promoter mutations that drive ectopic expression of GRHL2 or OVOL2 in corneal endothelial cells may result in cell state change through the mesenchymal-to-epithelial transition. GRHL2 activates OVOL2 expression such that ectopic expression of GRHL2 will also result in ectopic OVOL2 expression. OVOL2 in turn regulates the transcriptional program of human corneal epithelium cells by repressing expression of mesenchymal genes such as ZEB1 [Frisch et al., 2017]. This will drive corneal endothelium cells to become epithelial-like, stratified, and irregularly shaped cells. By transactivating expression of OVOL2, a gene associated with PPCD, GRHL2 in turn activates expression of Ecadherin, claudin 4 (epidermal tight junctions), and Rab25 (apical trafficking) [Boivin and Schmidt-Ott, 2017].

Disturbances of the delicate balance between ZEB1, OVOL2, and GRHL2 levels will thus result in either of 3 forms of PPCD [Liskova et al., 2018]. OVOL2 ectopic expression in corneal endothelial cells leads to high OVOL2/ low ZEB1 PPCD1. ZEB1 haploinsufficiency in corneal endothelial cells will produce low ZEB1/high OVOL2/ 
high GRHL2 PPCD3. Finally, GRHL2 ectopic expression will result in low ZEB1/high GRHL2 PPCD4. In view of the current information on human PPCD patients, this is a plausible model to explain 3 forms of PPCD, which merits testing in model organisms as has been done with the involvement of the $\mathrm{IHH}$ gene in relation to disturbed development of cranial and long bones.

These publications demonstrate the importance of CNVs and SNVs in noncoding genome segments and reemphasize the limitations of targeted sequencing and whole exome sequencing. Thus far, mutations in noncoding regions have been underestimated or even neglected in medical genetics. Intronic losses or duplications in developmental genes, such as ARHGEF39, BTN2A1, CNT-
NAP2, CENPJ, MTMR3, TRIP10, WWOX, and ZNF701, which may affect transcription and microRNA binding sites, or intragenic enhancers, prove to be more widespread and to have a larger impact than as yet appreciated [Cinghu et al., 2017; Poot, 2017; Devanna et al., 2018]. The publications discussed above also suggest that precisely balanced gene networks, governing the transition from one cell type to another, may be paramount for proper development. Ectopic gene expression, due to noncoding mutations and interfering with such regulatory networks, may become a novel paradigm in developmental genetics.

Martin Poot

\section{References}

-Aghajanian P, Xing W, Cheng S, Mohan S: Epiphyseal bone formation occurs via thyroid hormone regulation of chondrocyte to osteoblast transdifferentiation. Sci Rep 7:10432 (2017).

Boivin FJ, Schmidt-Ott KM: Transcriptional mechanisms coordinating tight junction assembly during epithelial differentiation. Ann NY Acad Sci 1397:80-99 (2017).

-Cartharius K, Frech K, Grote K, Klocke B, Haltmeier M, et al: MatInspector and beyond: promoter analysis based on transcription factor binding sites. Bioinformatics 21:2933-2942 (2005).

-Cinghu S, Yang P, Kosak JP, Conway AE, Kumar $\mathrm{D}$, et al: Intragenic enhancers attenuate host gene expression. Mol Cell 68:104-117 (2017).

Devanna P, Chen XS, Ho J, Gajewski D, Smith SD, et al: Next-gen sequencing identifies noncoding variation disrupting miRNA-binding sites in neurological disorders. Mol Psychiatry 23:1375-1384 (2018).

Frisch SM, Farris JC, Pifer PM: Roles of Grainyhead-like transcription factors in cancer. Oncogene 36:6067-6073 (2017).

Grabe N: AliBaba2: context specific identification of transcription factor binding sites. In Silico Biol 2:S1-15 (2002).

-Kim YR, Kim MA, Sagong B, Bae SH, Lee HJ, et al: Evaluation of the contribution of the EYA4 and GRHL2 genes in Korean patients with autosomal dominant non-syndromic hearing loss. PLoS One 10:e0119443 (2015).
Klopocki E, Lohan S, Brancati F, Koll R, Brehm A, et al: Copy-number variations involving the $\mathrm{IHH}$ locus are associated with syndactyly and craniosynostosis. Am J Hum Genet 88:70-75 (2011).

Krebs I, Weis I, Hudler M, Rommens JM, Roth H, et al: Translocation breakpoint maps $5 \mathrm{~kb} 3^{\prime}$ from TWIST in a patient affected with Saethre-Chotzen syndrome. Hum Mol Genet 6: 1079-1086 (1997).

Lenton K, James AW, Manu A, Brugmann SA, Birker D, et al: Indian hedgehog positively regulates calvarial ossification and modulates bone morphogenetic protein signaling. Genesis 49:784-796 (2011).

-Liskova P, Dudakova L, Evans CJ, Rojas Lopez KE, Pontikos N, et al: Ectopic GRHL2 expression due to non-coding mutations promotes cell state transition and causes posterior polymorphous corneal dystrophy 4. Am J Hum Genet 102:447-459 (2018).

Ohba S: Hedgehog signaling in endochondral ossification. J Dev Biol 4:E20 (2016).

$\checkmark$ Paumard-Hernández B, Berges-Soria J, Barroso E, Rivera-Pedroza CI, Pérez-Carrizosa V, et al: Expanding the mutation spectrum in 182 Spanish probands with craniosynostosis: identification and characterization of novel TCF12 variants. Eur J Hum Genet 23:907-914 (2015).
Petrof G, Nanda A, Howden J, Takeichi T, McMillan JR, et al: Mutations in GRHL2 result in an autosomal-recessive ectodermal Dysplasia syndrome. Am J Hum Genet 95: 308-314 (2014).

Poot M: Intragenic CNTNAP2 deletions: a bridge too far? Mol Syndromol 8:118-130 (2017).

Poot M: Structural genome variations related to craniosynostosis. Mol Syndromol, E-pub ahead of print (2018).

Van Laer L, Van Eyken E, Fransen E, Huyghe JR, Topsakal V, et al: The grainyhead like 2 gene (GRHL2), alias TFCP2L3, is associated with age-related hearing impairment. Hum Mol Genet 17:159-169 (2008).

Veistinen LK, Mustonen T, Hasan MR, Takatalo M, Kobayashi Y, et al: Regulation of calvarial osteogenesis by concomitant de-repression of GLI3 and activation of IHH targets. Front Physiol 18:1036 (2017).

Vona B, Nanda I, Neuner C, Müller T, Haaf T: Confirmation of GRHL2 as the gene for the DFNA28 locus. Am J Med Genet A 161A: 2060-2065 (2013).

Will AJ, Cova G, Osterwalder M, Chan WL, Wittler L, et al: Composition and dosage of a multipartite enhancer cluster control developmental expression of Ihh (Indian hedgehog). Nat Genet 49:1539-1545 (2017).

Yuksel-Apak M, Bögershausen N, Pawlik B, Li Y, Apak S, et al: A large duplication involving the IHH locus mimics acrocallosal syndrome. Eur J Hum Genet 20:639-644 (2012). 